\title{
CERGA Photoelectric astrolabe - Computational and instrumental improvements
}

\author{
B. Chauvineau, A. Journet, G. Vigouroux, J. Kovalevsky, \\ G. Billaud, R. Boche and M. Furia \\ CERGA - Observatoire de la Côte d'Azur \\ Avenue Copernic \\ F - 06130 Grasse, France
}

The compilation of three years of observations by the improved photoelectric astrolabe of CERGA has provided a star catalogue with an internal precision of 0.03 " in right ascension and 0.04" in declination for 47 stars and a similar precision for 82 other stars solved only for right ascension (Vigouroux et al., 1992). The classical group reduction was used. External comparisons with preliminary HIPPARCOS positions showed that these numbers represent the accuracy of the catalogue. However, many changes in the instrument and in the reduction procedures are contemplated that should bring new significant improvements in these results.

The instrument is being redesigned so as to reduce the main cause of thermal perturbations which is the presence of the concrete pillar on which both the instrument and the mercury mirror stand. A complex thermal situation develops within the instrument due to temperature differences that often are of the order of 3 degrees or more. The effect is to shift the images in an unpredictable manner. A new iron pillar, largely open to ambient air has been designed and is being built. The mercury mirror elevator will be installed in the pillar to allow observations at $30^{\circ}$ and $45^{\circ}$ zenith distances. These actions, and some other modifications prepare in addition a complete automatization of the instrument.

A new reduction procedure is being implemented. All observations covering one year or more are used simultaneously in order to determine not only stellar positions, but also many instrumental and local parameters. Among these are some seasonal terms in the prism angle, refraction corrections and temperatures measured in various critical points within the instrument. The recognition and preliminary evaluation of these parameters will be done using HIPPARCOS results for star positions.

\section{Reference}

Vigouroux, G., Billaud, G., Boche, R., Chauvineau, B., Furia, M., Journet, A. and Kovalevsky, J. (1992) Astron. and Astroph. Suppl. (in press). 